\title{
Vibration control for adjacent structures using local state information
}

\author{
F. Palacios-Quiñonero ${ }^{\mathrm{a}, *}$, J. Rubió-Massegúa ${ }^{\mathrm{a}}$ J. M. Rossell ${ }^{\mathrm{a}}$, H. R. Karimi ${ }^{\mathrm{b}}$ \\ ${ }^{a}$ Dept. of Applied Mathematics III, Universitat Politècnica de Catalunya (UPC), 08242-Manresa, Spain \\ ${ }^{b}$ Dept. of Engineering, Faculty of Engineering and Science, University of Agder (UiA), N-4898 Grimstad, Norway
}

\begin{abstract}
In this paper, a novel strategy for structural vibration control of multi-structure systems is presented. This strategy pays particular attention to mitigating negative interstructure interactions. Moreover, it is based on recent advances in static output-feedback control, which make possible the efficient computation of decentralized velocity-feedback controllers by solving a single-step optimization problem with Linear Matrix Inequality constraints. To illustrate the main ideas, a local velocity-feedback energyto-peak controller is designed for the seismic protection of a two-building system. This controller is remarkably effective and extremely simple. Moreover, it can also be implemented by a linear passive damper. To assess the effectiveness of the proposed controller, numerical simulations are conducted with positive results.
\end{abstract}

Keywords: structural vibration control, multi-structure systems, static output-feedback, passive damping design

\section{Introduction}

One of the main objectives of Structural Vibration Control (SVC) for large structures is to mitigate the vibrational response induced by external natural disturbances, such as wind gusts, earthquakes, or ocean waves. For multi-structure systems, the overall response must include not only the vibrational response of individual substructures, but also the possible interactions between adjacent substructures.

The seismic protection of closely adjacent buildings is an excellent example of SVC for multi-structure systems. In this case, the action of seismic excitations can produce interbuilding collisions (pounding), which can cause severe structural damage. Moreover, the large acceleration pulses generated in the quick and massive pounding impacts can also produce a serious damage in the buildings' content [1-5]. Consequently, a twofold objective must be considered in SVC designs for this kind of multi-structure systems: (1) mitigating the structural vibrational response of the individual buildings, and (2) providing protection against pounding events.

The Connected Control Method (CCM) consists in linking together adjacent buildings by coupling devices to produce appropriate reaction control forces. Over the last years, a number of passive, active, and semiactive control strategies based on the CCM approach have been proposed for seismic protection of adjacent buildings with positive results (see for example [615]). It should be highlighted, however, that all these works only deal with the vibrational response of the individual buildings.

\footnotetext{
*Corresponding author

Email addresses: francisco.palacios@upc.edu (F.

Palacios-Quiñonero), josep.rubio@upc.edu (J. Rubió-Massegú),

josep.maria.rossell@upc.edu (J. M. Rossell),

hamid.r.karimi@uia.no (H. R. Karimi )
}

An attempt of setting a more comprehensive formulation of the problem can be found in [16, 17], where two different kinds of output variables are considered. In these papers, together with the interstory drifts typically used to describe the relative displacement of adjacent stories in the same building, the interbuilding approaches are introduced to describe the approaching between stories placed at the same level in adjacent buildings.

In contrast with previous works, the present paper is principally focused on the interactions between adjacent buildings. More precisely, the main goal is to design a control system to provide a suitable protection against negative interbuilding interactions produced by seismic excitations. This should also be done without introducing negative side effects in the structural vibration response of the individual buildings. Moreover, the control system should be as simple as possible to facilitate its practical implementation. In terms of the output variables, these controller design objectives can be formulated as follows: (1) to produce a significant reduction of the interbuilding approach peak values, while (2) helping to keep the peak values of the interstory drifts in the individual buildings within acceptable levels. Additionally, the simplicity constraint is a broad concept which may involve a variety of different design elements, such as partial state information requirements, reduced information exchange, or low power consumption.

Decentralized velocity-feedback controllers can be efficiently designed using recent developments on static output-feedback control presented in [18]. This approach has been successfully applied to design decentralized velocity-feedback controllers and optimal passive-damping systems for seismic protection of single buildings $[19,20]$. In the present work, these new ideas are applied to design a local velocity-feedback energy-to-peak controller which satisfies the proposed design objectives.

For clarity and brevity, a particular two-building system formed by a four-story building adjacent to a five-story building 
has been selected to present the main ideas. A minimal actuation system has also been chosen, which consists in a single actuation device linking both buildings at the fourth story level, as schematically depicted in Fig. 1. For this two-building system, a velocity-feedback controller that only uses the relative velocity of the fourth stories as feedback information is designed. This controller attains a remarkable reduction of the interbuilding approach peak values and, also, a moderate attenuation of the interstory drift peak values in both buildings. Moreover, it can be implemented in practice using a linear passive damper, that is, without sensors, no communication system, and null power consumption. A state-feedback LQR controller and a state-feedback energy-to-peak controller, which require the complete two-building state as feedback information, are also computed and used as a reference.

To assess the effectiveness of the proposed controllers, numerical simulations are conducted using the full scale NorthSouth El Centro 1940 seismic record as ground acceleration disturbance. To avoid the computational complexity associated to the pounding impacts, the numerical simulations are carried out under the assumption that the interbuilding separation is large enough to avoid collisions. In this case, the maximum values of the interbuilding approaches can be understood as lower bounds of safe interbuilding separation.

The paper is organized as follows: In Section 2, a secondorder model and a first-order state-space model for the twobuilding system are provided. In Section 3, the theoretical results on static output-feedback control presented in [18] are applied to derive an effective computational strategy to design static output-feedback energy-to-peak controllers. In Section 4, the different controllers are computed and numerical simulations are conducted to compare their effectiveness. Finally, some conclusions and future research directions are presented in Section 5.

\section{Two-building mathematical model}

\subsection{Second-order model}

Let us consider the two-building system schematically displayed in Fig. 1. The buildings motion can be described by the second-order model

$$
\mathbf{M} \ddot{\mathbf{q}}(t)+\mathbf{C} \dot{\mathbf{q}}(t)+\mathbf{K q}(t)=\mathbf{T}_{u} u(t)+\mathbf{T}_{w} w(t),
$$

where $\mathbf{M}$ is the mass matrix, $\mathbf{C}$ is the damping matrix, and $\mathbf{K}$ is the stiffness matrix. The vector of story displacements with respect to the ground is

$$
\mathbf{q}(t)=\left[\begin{array}{l}
\mathbf{q}^{(1)}(t) \\
\mathbf{q}^{(2)}(t)
\end{array}\right],
$$

where

$$
\begin{gathered}
\mathbf{q}^{(1)}(t)=\left[q_{1}^{1}(t), q_{2}^{1}(t), q_{3}^{1}(t), q_{4}^{1}(t)\right]^{T}, \\
\mathbf{q}^{(2)}(t)=\left[q_{1}^{2}(t), q_{2}^{2}(t), q_{3}^{2}(t), q_{4}^{2}(t), q_{5}^{2}(t)\right]^{T},
\end{gathered}
$$

and $q_{i}^{j}(t)$ represents the displacement of the $i$ th story in the $j$ th building corresponding to the time $t$. We assume that an active

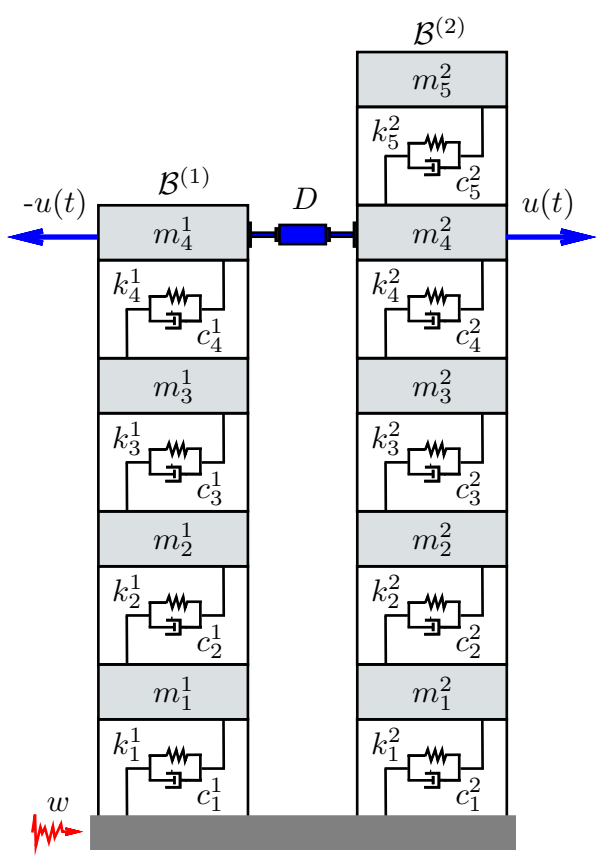

Figure 1: Two-building system with interbuilding actuation device

control device $D$ has been implemented between the fourth stories of both structures. The control force $u(t)$ delivered by $D$ produces a pair of opposite forces as indicated in Fig. 1. This actuation scheme is modeled by means of the control location matrix $\mathbf{T}_{u}$. Finally, the ground acceleration disturbance is denoted by $w(t)$, and $\mathbf{T}_{w}$ is the disturbance input matrix. The mass matrix $\mathbf{M}$ has the following block diagonal structure:

$$
\mathbf{M}=\left[\begin{array}{cc}
\mathbf{M}^{(1)} & {[\mathbf{0}]_{4 \times 5}} \\
{[\mathbf{0}]_{5 \times 4}} & \mathbf{M}^{(2)}
\end{array}\right],
$$

where $[\mathbf{0}]_{r \times s}$ is a zero matrix of dimensions $r \times s$,

$$
\begin{gathered}
\mathbf{M}^{(1)}=\left[\begin{array}{cccc}
m_{1}^{1} & 0 & 0 & 0 \\
0 & m_{2}^{1} & 0 & 0 \\
0 & 0 & m_{3}^{1} & 0 \\
0 & 0 & 0 & m_{4}^{1}
\end{array}\right], \\
\mathbf{M}^{(2)}=\left[\begin{array}{ccccc}
m_{1}^{2} & 0 & 0 & 0 & 0 \\
0 & m_{2}^{2} & 0 & 0 & 0 \\
0 & 0 & m_{3}^{2} & 0 & 0 \\
0 & 0 & 0 & m_{4}^{2} & 0 \\
0 & 0 & 0 & 0 & m_{5}^{2}
\end{array}\right],
\end{gathered}
$$

and $m_{i}^{j}$ denotes the mass of the $i$ th story in the $j$ th building. The stiffness matrix has the form

$$
\mathbf{K}=\left[\begin{array}{cc}
\mathbf{K}^{(1)} & {[\mathbf{0}]_{4 \times 5}} \\
{[\mathbf{0}]_{5 \times 4}} & \mathbf{K}^{(2)}
\end{array}\right],
$$

where

$$
\mathbf{K}^{(1)}=\left[\begin{array}{cccc}
k_{1}^{1}+k_{2}^{1} & -k_{2}^{1} & 0 & 0 \\
-k_{2}^{1} & k_{2}^{1}+k_{3}^{1} & -k_{3}^{1} & 0 \\
0 & -k_{3}^{1} & k_{3}^{1}+k_{4}^{1} & -k_{4}^{1} \\
0 & 0 & -k_{4}^{1} & k_{4}^{1}
\end{array}\right]
$$




$$
\mathbf{K}^{(2)}=\left[\begin{array}{ccccc}
k_{1}^{2}+k_{2}^{2} & -k_{2}^{2} & 0 & 0 & 0 \\
-k_{2}^{2} & k_{2}^{2}+k_{3}^{2} & -k_{3}^{2} & 0 & 0 \\
0 & -k_{3}^{2} & k_{3}^{2}+k_{4}^{2} & -k_{4}^{2} & 0 \\
0 & 0 & -k_{4}^{2} & k_{4}^{2}+k_{5}^{2} & -k_{5}^{2} \\
0 & 0 & 0 & -k_{5}^{2} & k_{5}^{2}
\end{array}\right],
$$

and $k_{i}^{j}$ denotes the stiffness coefficient of the $i$ th story in the $j$ th building. The damping matrix also has a block diagonal structure of the form

$$
\mathbf{C}=\left[\begin{array}{cc}
\mathbf{C}^{(1)} & {[\mathbf{0}]_{4 \times 5}} \\
{[\mathbf{0}]_{5 \times 4}} & \mathbf{C}^{(2)}
\end{array}\right] .
$$

When the damping coefficients are known, the matrices $\mathbf{C}^{(1)}$ and $\mathbf{C}^{(2)}$ can be obtained by replacing the stiffness coefficients $k_{i}^{j}$ in Eqs. (9) and (10) by the corresponding damping coefficients $c_{i}^{j}$. Frequently, however, the values of the damping coefficients cannot be properly determined and the matrices $\mathbf{C}^{(1)}$ and $\mathbf{C}^{(2)}$ are computed following other methods such as the Rayleigh damping approach [21]. The control location matrix is

$$
\mathbf{T}_{u}=\left[\begin{array}{lllllllll}
0 & 0 & 0 & -1 & 0 & 0 & 0 & 1 & 0
\end{array}\right]^{T}
$$

and the disturbance input matrix can be written as

$$
\mathbf{T}_{w}=-\mathbf{M}[\mathbf{1}]_{9 \times 1},
$$

where $[\mathbf{1}]_{9 \times 1}$ is a column vector of dimension 9 with all its entries equal to 1 .

In the different controller designs and numerical simulations presented in Section 4, the following particular mass values $\left(\times 10^{3} \mathrm{~kg}\right)$ have been used: $m_{1}^{1}=215.2, m_{2}^{1}=209.2, m_{3}^{1}=$ 207.0, $m_{4}^{1}=266.1 ; m_{1}^{2}=215.2, m_{2}^{2}=209.2, m_{3}^{2}=207.0, m_{4}^{2}=$ 204.8, $m_{5}^{2}=266.1$. The particular values of the stiffness coefficients $\left(\times 10^{6} \mathrm{~N} / \mathrm{m}\right)$ are: $k_{1}^{1}=147, k_{2}^{1}=113, k_{3}^{1}=99, k_{4}^{1}=84$; $k_{1}^{2}=147, k_{2}^{2}=113, k_{3}^{2}=99, k_{4}^{2}=89, k_{5}^{2}=84$. These values of mass and stiffness coefficients are similar to those corresponding to the five-story building presented in [22]. The matrices $\mathbf{C}^{(1)}$ and $\mathbf{C}^{(2)}$ have been computed as Rayleigh damping matrices by setting a $2 \%$ of relative damping on the corresponding first and last modes. The obtained particular values (in Ns/m) are as follows:

$$
\begin{gathered}
\mathbf{C}^{(1)}=10^{5} \times\left[\begin{array}{cccc}
2.6450 & -0.9034 & 0 & 0 \\
-0.9034 & 2.2455 & -0.7915 & 0 \\
0 & -0.7915 & 2.7078 & -0.6715 \\
0 & 0 & -0.6715 & 1.3719
\end{array}\right], \\
\mathbf{C}^{(2)}=10^{5} \times\left[\begin{array}{ccccc}
2.6017 & -0.9244 & 0 & 0 & 0 \\
-0.9244 & 2.1958 & -0.8099 & 0 & 0 \\
0 & -0.8099 & 1.9946 & -0.7281 & 0 \\
0 & 0 & -0.7281 & 1.8670 & -0.6872 \\
0 & 0 & 0 & -0.6872 & 1.2741
\end{array}\right] .
\end{gathered}
$$

\subsection{First-order state-space model}

Now we consider the first-order state-space model

$$
\dot{\mathbf{x}}(t)=\mathbf{A} \mathbf{x}(t)+\mathbf{B} u(t)+\mathbf{E} w(t),
$$

with state vector

$$
\mathbf{x}(t)=\left[\begin{array}{c}
\mathbf{q}(t) \\
\dot{\mathbf{q}}(t)
\end{array}\right]
$$

The state matrix in Eq. (16) can be written as

$$
\mathbf{A}=\left[\begin{array}{cc}
{[\mathbf{0}]_{9 \times 9}} & \mathbf{I}_{9} \\
-\mathbf{M}^{-1} \mathbf{K} & -\mathbf{M}^{-1} \mathbf{C}
\end{array}\right],
$$

where $\mathbf{I}_{r}$ denotes the identity matrix of order $r$. The control and disturbance input matrices have, respectively, the following form:

$$
\mathbf{B}=\left[\begin{array}{c}
{[\mathbf{0}]_{9 \times 1}} \\
\mathbf{M}^{-1} \mathbf{T}_{u}
\end{array}\right], \quad \mathbf{E}=\left[\begin{array}{r}
{[\mathbf{0}]_{9 \times 1}} \\
-[\mathbf{1}]_{9 \times 1}
\end{array}\right] .
$$

In addition to the state variables, two different sets of output variables are considered in this work: interstory drifts and interbuilding approaches. The interstory drifts are the relative displacements between consecutive floors of the same building, and can be defined as

$$
\left\{\begin{array}{l}
s_{1}^{j}(t)=q_{1}^{j}(t), \\
s_{i}^{j}(t)=q_{i}^{j}(t)-q_{i-1}^{j}(t), \quad 1<i \leq n_{j},
\end{array}\right.
$$

where $n_{j}$ represents the number of stories of building $j$. For building $\mathcal{B}^{(1)}$, the vector of interstory drifts is

$$
\mathbf{s}^{(1)}(t)=\left[s_{1}^{1}(t), s_{2}^{1}(t), s_{3}^{1}(t), s_{4}^{1}(t)\right]^{T},
$$

and for $\mathcal{B}^{(2)}$, we have

$$
\mathbf{s}^{(2)}(t)=\left[s_{1}^{2}(t), s_{2}^{2}(t), s_{3}^{2}(t), s_{4}^{2}(t), s_{5}^{2}(t)\right]^{T} .
$$

The overall vector of interstory drifts

$$
\mathbf{s}(t)=\left[\begin{array}{l}
\mathbf{s}^{(1)}(t) \\
\mathbf{s}^{(2)}(t)
\end{array}\right]
$$

can be computed as

$$
\mathbf{s}(t)=\mathbf{C}_{s} \mathbf{x}(t)
$$

with the output matrix

$$
\left.\mathbf{C}_{s}=\left[\begin{array}{cc}
\tilde{\mathbf{C}}_{s} & {[\mathbf{0}}
\end{array}\right]_{9 \times 9}\right],
$$

where

$$
\begin{gathered}
\tilde{\mathbf{C}}_{s}=\left[\begin{array}{ccc}
\mathbf{C}_{s}^{(1)} & {[\mathbf{0}]_{4 \times 5}} \\
{[\mathbf{0}]_{5 \times 4}} & \mathbf{C}_{s}^{(2)}
\end{array}\right], \\
\mathbf{C}_{s}^{(1)}=\left[\begin{array}{rrrrr}
1 & 0 & 0 & 0 \\
-1 & 1 & 0 & 0 \\
0 & -1 & 1 & 0 \\
0 & 0 & -1 & 1
\end{array}\right], \\
\mathbf{C}_{s}^{(2)}=\left[\begin{array}{rrrrr}
1 & 0 & 0 & 0 & 0 \\
-1 & 1 & 0 & 0 & 0 \\
0 & -1 & 1 & 0 & 0 \\
0 & 0 & -1 & 1 & 0 \\
0 & 0 & 0 & -1 & 1
\end{array}\right] .
\end{gathered}
$$

The interbuilding approaches describe the approaching between the stories placed at the same level in adjacent buildings

$$
a_{i}(t)=-\left(q_{i}^{2}(t)-q_{i}^{1}(t)\right), 1 \leq i \leq \min \left(n_{1}, n_{2}\right) .
$$


For our particular two-building system, the vector of interbuilding approaches

$$
\mathbf{a}(t)=\left[a_{1}(t), a_{2}(t), a_{3}(t), a_{4}(t)\right]^{T},
$$

can be computed as

$$
\mathbf{a}(t)=\mathbf{C}_{a} \mathbf{x}(t),
$$

using the output matrix

$$
\left.\mathbf{C}_{a}=\left[\begin{array}{ll}
\tilde{\mathbf{C}}_{a} & {[\mathbf{0}}
\end{array}\right]_{4 \times 9}\right],
$$

where

$$
\left.\tilde{\mathbf{C}}_{a}=\left[\begin{array}{lll}
\mathbf{I}_{4} & -\mathbf{I}_{4} & {[\mathbf{0}}
\end{array}\right]_{4 \times 1}\right] .
$$

Remark 1. It should be observed that positive values of the interbuilding approaches defined in Eq. (29) correspond to a reduction of the distance between the corresponding stories. Clearly, for a given interbuilding separation, large values of the interbuilding approaches may result in interbuilding collisions.

\section{Static output-feedback energy-to-peak controller design}

In this section, the theoretical results presented in [18] are applied to define an effective computational strategy to design static output-feedback energy-to-peak controllers. We begin by considering the system

$$
\mathcal{S}:\left\{\begin{array}{l}
\dot{\mathbf{x}}(t)=\mathbf{A} \mathbf{x}(t)+\mathbf{B} \mathbf{u}(t)+\mathbf{E} \mathbf{w}(t) \\
\mathbf{z}(t)=\mathbf{C}_{z} \mathbf{x}(t)+\mathbf{D}_{z} \mathbf{u}(t)
\end{array}\right.
$$

where $\mathbf{x}(t) \in \mathbb{R}^{n}$ is the state, $\mathbf{u}(t) \in \mathbb{R}^{m}$ is the control input, $\mathbf{w}(t) \in \mathbb{R}^{r}$ is the disturbance input, and $\mathbf{z}(t) \in \mathbb{R}^{n_{z}}$ is the controlled output. $\mathbf{A}, \mathbf{B}, \mathbf{E}, \mathbf{C}_{z}$, and $\mathbf{D}_{z}$ are known, real and constant matrices of appropriate dimensions. Given a state-feedback controller

$$
\mathbf{u}(t)=\mathbf{G} \mathbf{x}(t),
$$

the following closed-loop system results:

$$
\mathcal{S}_{C L}:\left\{\begin{array}{l}
\dot{\mathbf{x}}(t)=\mathbf{A}_{G} \mathbf{x}(t)+\mathbf{E} \mathbf{w}(t) \\
\mathbf{z}(t)=\mathbf{C}_{G} \mathbf{x}(t)
\end{array}\right.
$$

where

$$
\mathbf{A}_{G}=\mathbf{A}+\mathbf{B ~ G}, \quad \mathbf{C}_{G}=\mathbf{C}_{z}+\mathbf{D}_{z} \mathbf{G} .
$$

The closed-loop transfer function from the disturbance $\mathbf{w}(t)$ to the controlled output $\mathbf{z}(t)$ is

$$
\mathbf{T}_{G}(s)=\mathbf{C}_{G}\left(s \mathbf{I}-\mathbf{A}_{G}\right)^{-1} \mathbf{E} .
$$

In the state-feedback energy-to-peak control design, the objective is to find a control gain matrix $\tilde{\mathbf{G}}$ which produces an asymptotically stable closed-loop matrix $\mathbf{A}_{\tilde{G}}$ and, at the same time, minimizes the value of the energy-to-peak norm

$$
\gamma_{G}=\left\|\mathbf{T}_{G}\right\|_{2, \infty}=\sup _{0<\|\mathbf{w}\|_{2}<\infty} \frac{\|\mathbf{z}\|_{\infty}}{\|\mathbf{w}\|_{2}}
$$

where

$$
\begin{gathered}
\|\mathbf{z}\|_{\infty}=\sup _{0 \leq t<\infty} \sqrt{\mathbf{z}^{T}(t) \mathbf{z}(t)}, \\
\|\mathbf{w}\|_{2}=\left(\int_{0}^{\infty} \mathbf{w}^{T}(t) \mathbf{w}(t) d t\right)^{1 / 2} .
\end{gathered}
$$

For a prescribed $\gamma>0$, the following two statements are equivalent [23-25]:

1. $\mathbf{A}_{G}$ is asymptotically stable, and $\left\|\mathbf{T}_{G}\right\|_{2, \infty}<\gamma$.

2. There exists a symmetric positive-definite matrix $\mathbf{X}$ such that

$$
\mathbf{A}_{G} \mathbf{X}+\mathbf{X} \mathbf{A}_{G}^{T}+\mathbf{E} \mathbf{E}^{T}<0, \quad \mathbf{C}_{G} \mathbf{X C}_{G}^{T}<\gamma^{2} \mathbf{I} .
$$

Using the closed-loop matrix definitions given in Eq. (37), the conditions in Eq. (42) become

$$
\begin{gathered}
(\mathbf{A}+\mathbf{B} \mathbf{G}) \mathbf{X}+\mathbf{X}(\mathbf{A}+\mathbf{B} \mathbf{G})^{T}+\mathbf{E} \mathbf{E}^{T}<0, \\
\left(\mathbf{C}_{z}+\mathbf{D}_{z} \mathbf{G}\right) \mathbf{X}\left(\mathbf{C}_{z}+\mathbf{D}_{z} \mathbf{G}\right)^{T}<\gamma^{2} \mathbf{I} .
\end{gathered}
$$

By introducing the new variables $\mathbf{Y}=\mathbf{G X}, \eta=\gamma^{2}$, and using Schur complements in Eq. (44), the nonlinear matrix inequalities in Eqs. (43) and (44) can be written as the following Linear Matrix Inequalities (LMIs):

$$
\begin{gathered}
\mathbf{A X}+\mathbf{X} \mathbf{A}^{T}+\mathbf{B Y}+\mathbf{Y}^{T} \mathbf{B}^{T}+\mathbf{E} \mathbf{E}^{T}<0 \\
{\left[\begin{array}{cc}
\mathbf{X} & \left(\mathbf{C}_{z} \mathbf{X}+\mathbf{D}_{z} \mathbf{Y}\right)^{T} \\
\mathbf{C}_{z} \mathbf{X}+\mathbf{D}_{z} \mathbf{Y} & \eta \mathbf{I}
\end{array}\right]>0}
\end{gathered}
$$

and the continuous-time state-feedback energy-to-peak control problem can be transformed into the following optimization problem with LMI constraints:

$\left\{\begin{array}{l}\text { minimize } \eta \\ \text { subject to } \mathbf{X}>0, \eta>0, \text { and the LMIs in Eqs. (45), (46), }\end{array}\right.$

where matrices $\mathbf{X}$ and $\mathbf{Y}$ are the optimization variables. If the optimal value $\tilde{\eta}$ is attained for the matrices $\tilde{\mathbf{X}}$ and $\tilde{\mathbf{Y}}$, then the control gain matrix

$$
\tilde{\mathbf{G}}=\tilde{\mathbf{Y}}(\tilde{\mathbf{X}})^{-1}
$$

defines a state-feedback controller $\mathbf{u}(t)=\tilde{\mathbf{G}} \mathbf{x}(t)$ with asymptotically stable closed-loop matrix $\mathbf{A}_{\tilde{G}}$ and optimal energy-to-peak norm

$$
\gamma_{\tilde{G}}=\left\|\mathbf{T}_{\tilde{G}}\right\|_{2, \infty}=\tilde{\eta}^{1 / 2} .
$$

Now, let us focus our attention on a more realistic scenario, where only a restricted set of observed output variables are available as feedback information. More precisely, we consider the observed output vector

$$
\mathbf{y}(t)=\mathbf{C}_{y} \mathbf{x}(t),
$$

where $\mathbf{y}(t) \in \mathbb{R}^{p}, p<n$, and $\mathbf{C}_{y}$ is a full row-rank matrix of dimensions $p \times n$. Following the ideas presented in [18], a static output-feedback energy-to-peak controller

$$
\mathbf{u}(t)=\mathbf{G}_{y} \mathbf{y}(t),
$$




$$
\begin{aligned}
& \mathbf{A} \mathbf{Q} \mathbf{X}_{Q} \mathbf{Q}^{T}+\mathbf{Q} \mathbf{X}_{Q} \mathbf{Q}^{T} \mathbf{A}^{T}+\mathbf{A} \mathbf{R} \mathbf{X}_{R} \mathbf{R}^{T}+\mathbf{R} \mathbf{X}_{R} \mathbf{R}^{T} \mathbf{A}^{T}+\mathbf{B} \mathbf{Y}_{R} \mathbf{R}^{T}+\mathbf{R} \mathbf{Y}_{R}^{T} \mathbf{B}^{T}+\mathbf{E} \mathbf{E}^{T}<0, \quad \text { (LMI-A) } \\
& {\left[\begin{array}{cc}
\mathbf{Q} \mathbf{X}_{Q} \mathbf{Q}^{T}+\mathbf{R} \mathbf{X}_{R} \mathbf{R}^{T} & \left(\mathbf{C}_{z} \mathbf{Q} \mathbf{X}_{Q} \mathbf{Q}^{T}+\mathbf{C}_{z} \mathbf{R} \mathbf{X}_{R} \mathbf{R}^{T}+\mathbf{D}_{z} \mathbf{Y}_{R} \mathbf{R}^{T}\right)^{T} \\
\mathbf{C}_{z} \mathbf{Q} \mathbf{X}_{Q} \mathbf{Q}^{T}+\mathbf{C}_{z} \mathbf{R} \mathbf{X}_{R} \mathbf{R}^{T}+\mathbf{D}_{z} \mathbf{Y}_{R} \mathbf{R}^{T} & \eta \mathbf{I}
\end{array}\right]>0}
\end{aligned}
$$

Figure 2: LMIs for static output-feedback energy-to-peak controller design

can be computed by defining the transformations

$$
\mathbf{X}=\mathbf{Q} \mathbf{X}_{Q} \mathbf{Q}^{T}+\mathbf{R} \mathbf{X}_{R} \mathbf{R}^{T}, \quad \mathbf{Y}=\mathbf{Y}_{R} \mathbf{R}^{T},
$$

where $\mathbf{R}$ is the $\mathbf{C}_{y}$ Moore-Penrose pseudo-inverse

$$
\mathbf{R}=\mathbf{C}_{y}^{T}\left(\mathbf{C}_{y} \mathbf{C}_{y}^{T}\right)^{-1}
$$

$\mathbf{Q}$ is a matrix with dimensions $n \times(n-p)$, whose columns are a basis of $\operatorname{Ker}\left(\mathbf{C}_{y}\right) ; \mathbf{X}_{Q}, \mathbf{X}_{R}$ are symmetric matrices with respective dimensions $(n-p) \times(n-p)$, and $p \times p$; and $\mathbf{Y}_{R}$ is an $m \times p$ matrix. After substituting the transformations given in Eq. (52) in the LMIs presented in Eqs. (45) and (46), we obtain the new set of LMIs displayed in Fig. 2; and the continuoustime static output-feedback energy-to-peak control problem can now be formulated as the following optimization problem with LMI constraints:

$$
\left\{\begin{array}{l}
\text { minimize } \eta \\
\text { subject to } \mathbf{X}_{Q}>0, \mathbf{X}_{R}>0, \eta>0, \text { and the LMIs in Fig. } 2
\end{array}\right.
$$

where matrices $\mathbf{X}_{Q}, \mathbf{X}_{R}$, and $\mathbf{Y}_{R}$ are the new optimization variables. If an optimal value $\tilde{\eta}$ is attained for the matrices $\tilde{\mathbf{X}}_{Q}, \tilde{\mathbf{X}}_{R}$, and $\tilde{\mathbf{Y}}_{R}$, then the control gain matrix

$$
\tilde{\mathbf{G}}_{y}=\tilde{\mathbf{Y}}_{R}\left(\tilde{\mathbf{X}}_{R}\right)^{-1}
$$

defines a static output-feedback controller $\mathbf{u}(t)=\tilde{\mathbf{G}}_{y} \mathbf{y}(t)$ with asymptotically stable closed-loop matrix

$$
\mathbf{A}_{\tilde{G}_{y}}=\mathbf{A}+\mathbf{B} \tilde{\mathbf{G}}_{y} \mathbf{C}_{y}
$$

and optimal energy-to-peak norm

$$
\gamma_{\tilde{G}_{y}}=\left\|\mathbf{T}_{\tilde{G}_{y}}\right\|_{2, \infty} \leq \tilde{\eta}^{1 / 2} .
$$

Remark 2. As indicated in Eq. (57), solving the optimization problem given in Eq. (54) only provides an upper bound of the $\gamma$-value corresponding to the controller defined by the outputfeedback control gain matrix $\tilde{\mathbf{G}}_{y}$. The actual value of $\gamma_{\tilde{G}_{y}}$ can be computed by considering the associated state-feedback gain matrix

$$
\hat{\mathbf{G}}=\tilde{\mathbf{G}}_{y} \mathbf{C}_{y}
$$

and the LMIs

$$
\begin{gathered}
(\mathbf{A}+\mathbf{B} \hat{\mathbf{G}}) \mathbf{X}+\mathbf{X}(\mathbf{A}+\mathbf{B} \hat{\mathbf{G}})^{T}+\mathbf{E} \mathbf{E}^{T}<0 \\
\left(\mathbf{C}_{z}+\mathbf{D}_{z} \hat{\mathbf{G}}\right) \mathbf{X}\left(\mathbf{C}_{z}+\mathbf{D}_{z} \hat{\mathbf{G}}\right)^{T}-\eta \mathbf{I}<0
\end{gathered}
$$

If the optimization problem

$\left\{\begin{array}{l}\text { minimize } \eta \\ \text { subject to } \mathbf{X}>0, \eta>0, \text { and the LMIs in Eqs. }(59),(60)\end{array}\right.$

admits the optimal solution $\hat{\eta}$, then we have

$$
\gamma_{\tilde{G}_{y}}=\hat{\eta}^{1 / 2} .
$$

Note that, in contrast with what happened in Eqs. (43) and (44), $\hat{\mathbf{G}}$ is a known matrix in Eqs. (59) and (60); consequently, this last pair of matrix inequalities are linear.

\section{Results and discussion}

\subsection{Controllers design}

In this subsection, the controller design methodology discussed in Section 3 is applied to compute a local velocity-feedback controller for the two-building system introduced in Section 2. A state-feedback LQR controller and a state-feedback energyto-peak controller are also computed to be used as a reference.

\subsubsection{State-feedback LQR controller}

Let us consider the state vector $\mathbf{x}(t)$ given in Eq. (17) and the matrix

$$
\hat{\mathbf{Q}}=\alpha_{s}^{2} \mathbf{C}_{s}^{T} \mathbf{C}_{s}+\alpha_{a}^{2} \mathbf{C}_{a}^{T} \mathbf{C}_{a},
$$

where $\mathbf{C}_{s}$ is the output matrix of interstory drifts defined in Eqs. (25)-(28), $\mathbf{C}_{a}$ is the output matrix of interbuilding approaches given in Eqs. (32) and (33), and $\alpha_{s}, \alpha_{a}$ are real scaling coefficients. The quadratic form

$$
\mathbf{x}^{T}(t) \hat{\mathbf{Q}} \mathbf{x}(t)=\alpha_{s}^{2} \sum_{j=1}^{2} \sum_{i=1}^{n_{j}}\left\{s_{i}^{j}(t)\right\}^{2}+\alpha_{a}^{2} \sum_{i=1}^{4}\left\{a_{i}(t)\right\}^{2}
$$

provides a joint quadratic cost of interstory drifts and interbuilding approaches. To compute a state-feedback LQR controller

$$
u(t)=\mathbf{G} \mathbf{x}(t),
$$

we take the state-space model

$$
\dot{\mathbf{x}}(t)=\mathbf{A} \mathbf{x}(t)+\mathbf{B} u(t),
$$

with the matrices A and B given in Eqs. (18) and (19), and the quadratic cost function

$$
J(\mathbf{x}(t), u(t))=\int_{0}^{\infty} \mathbf{x}^{T}(t) \hat{\mathbf{Q}} \mathbf{x}(t)+\hat{R}\{u(t)\}^{2} d t .
$$




\begin{tabular}{|c|c|c|c|c|c|c|c|c|c|}
\hline$\tilde{\mathbf{G}}_{I}=10^{6} \times[$ & $\begin{array}{c}0.1391 \\
-0.0174\end{array}$ & $\begin{array}{c}0.4379 \\
-0.0475\end{array}$ & $\begin{array}{c}-0.3536 \\
0.0108\end{array}$ & $\begin{array}{l}3.3958 \\
0.9262\end{array}$ & $\begin{array}{c}-0.0890 \\
0.0212\end{array}$ & $\begin{array}{c}0.6150 \\
-0.0385\end{array}$ & $\begin{array}{c}2.0755 \\
-0.1707\end{array}$ & $\begin{array}{l}-4.3886 \\
-0.8512\end{array}$ & $\begin{array}{c}2.6393 \\
-0.1954]\end{array}$ \\
\hline & 0015 & $\begin{array}{l}0.303 \\
0.027\end{array}$ & $\begin{array}{l}0.5749 \\
0.0364\end{array}$ & 0.0502 & $\begin{array}{l}-0.3742 \\
-0.0194\end{array}$ & $\begin{array}{l}-0.1379 \\
-0.0489\end{array}$ & $\begin{array}{l}-0.0133 \\
-0.0820\end{array}$ & $\begin{array}{l}-0.1517 \\
-0.1106\end{array}$ & $\begin{array}{c}0.3113 \\
-0.1704\end{array}$ \\
\hline
\end{tabular}

Figure 3: Control gain matrices for the state-feedback LQR controller and the state-feedback energy-to-peak controller

The control gain matrix that minimizes the index in Eq. (67), subject to the constraints given in Eqs. (65) and (66), can be easily computed with the $\operatorname{lqr}()$ command of the MATLAB Control System Toolbox [26]. In Fig. 3, we present the optimal solution $\tilde{\mathbf{G}}_{I}$ corresponding to the particular values of the buildings parameters given in Subsection 2.1, the weighting matrix $\hat{\mathbf{Q}}$ with scaling coefficients $\alpha_{s}=5, \alpha_{a}=1$, and the weighting factor $\hat{R}=10^{-13}$.

Remark 3. Typically, the peak values of interbuilding approaches are significantly larger than those observed in interstory drifts. The scaling coefficients $\alpha_{s}$ and $\alpha_{a}$ in Eq. (63) are introduced to compensate for this effect, which can be clearly appreciated in the graphics presented in Subsection 4.2 (see also the graphics in $[16,17])$.

Remark 4. Note that the state-feedback LQR control gain matrix $\tilde{\mathbf{G}}_{I}$ is a row matrix of dimensions $1 \times 18$. For clarity, however, it has been presented in the figure using a two-row layout.

\subsubsection{State-feedback energy-to-peak controller}

In this second case, we consider the state-space model

$$
\dot{\mathbf{x}}(t)=\mathbf{A} \mathbf{x}(t)+\mathbf{B} u(t)+\mathbf{E} w(t)
$$

with the matrices A, B, E given in Eqs. (18) and (19), and the controlled output

$$
\mathbf{z}(t)=\mathbf{C}_{z} \mathbf{x}(t)+\mathbf{D}_{z} u(t)
$$

defined by the matrices

$$
\begin{gathered}
\mathbf{C}_{z}=\left[\begin{array}{cc}
\tilde{\mathbf{Q}}^{1 / 2} & {[\mathbf{0}]_{9 \times 9}} \\
{[\mathbf{0}]_{1 \times 9}} & {[\mathbf{0}]_{1 \times 9}}
\end{array}\right], \\
\mathbf{D}_{z}=\left[\begin{array}{c}
{[\mathbf{0}]_{9 \times 1}} \\
\tilde{R}
\end{array}\right],
\end{gathered}
$$

where

$$
\tilde{\mathbf{Q}}=\tilde{\alpha}_{s}^{2} \tilde{\mathbf{C}}_{s}^{T} \tilde{\mathbf{C}}_{s}+\tilde{\alpha}_{a}^{2} \tilde{\mathbf{C}}_{a}^{T} \tilde{\mathbf{C}}_{a}
$$

and the matrices $\tilde{\mathbf{C}}_{s}, \tilde{\mathbf{C}}_{a}$ are given in Eqs. (26) and (33).

As indicated in Section 3, a state-feedback energy-to-peak controller with the form given in Eq. (65) can be computed by solving the optimization problem with LMI constraints defined in Eq. (47). Solving this problem with the optimization tools of the MATLAB Robust Optimization Toolbox [27] for the particular values of the buildings parameters given in Subsection 2.1, and

$$
\tilde{\alpha}_{s}=5, \quad \tilde{\alpha}_{a}=1, \quad \tilde{R}=10^{-6.5}
$$

produces the control gain matrix $\tilde{\mathbf{G}}_{I I}$ displayed in Fig. 3, with an associated $\gamma$-value

$$
\gamma_{\tilde{G}_{I I}}=0.3905
$$

Remark 5. The controlled output $\mathbf{z}(t)$ corresponding to the particular values

$$
\tilde{\alpha}_{s}=\alpha_{s}, \quad \tilde{\alpha}_{a}=\alpha_{a}, \quad \tilde{R}=\hat{R}^{1 / 2}
$$

satisfies

$$
\mathbf{z}^{T}(t) \mathbf{z}(t)=\mathbf{x}^{T}(t) \hat{\mathbf{Q}} \mathbf{x}(t)+\hat{R}\{u(t)\}^{2},
$$

where $\hat{\mathbf{Q}}$ and $\hat{R}$ are the weighting matrices used in the quadratic index defined in Eq. (67). This choice of the controlled output produces a relatively similar behavior of the controllers designed following the LQR and energy-to-peak approach.

Remark 6. A complete knowledge of the state variables is required to compute the control actions with the state-feedback energy-to-peak control gain matrix $\tilde{\mathbf{G}}_{I I}$. Consequently, a full set of sensors and a complex communication system would be necessary for a practical implementation of the corresponding control system. This remark also applies to the controller defined by the control gain matrix $\tilde{\mathbf{G}}_{\boldsymbol{I}}$.

\subsubsection{Energy-to-peak controller with restricted local state in- formation}

Now, let us assume that the information available for feedback purposes is reduced to the relative velocity between the stories at the fourth level of the buildings. To design this third controller, we consider the same state-space model and controlled output used in Subsection 4.1.2, and define the observed output variable

$$
y(t)=\dot{q}_{4}^{2}(t)-\dot{q}_{4}^{1}(t),
$$

which can be written as

$$
y(t)=\mathbf{C}_{y} \mathbf{x}(t)
$$

with

$$
\mathbf{C}_{y}=[0,0,0,0,0,0,0,0,0,0,0,0,-1,0,0,0,1,0] .
$$

Next, we compute the matrices that define the transformation of the LMI variables presented in Eq. (52). The particular values of the matrices $\mathbf{Q}$ and $\mathbf{R}$ used in the controller design are displayed in Fig. 4. These matrices have been obtained with the MATLAB commands null( ) and pinv( ), respectively. Note that an orthonormal basis of $\operatorname{Ker}\left(\mathbf{C}_{y}\right)$ is provided by the null ( ) command. 


$$
\mathbf{Q}=\left[\begin{array}{ccccccccccccccccc}
0 & 0 & 0 & 0 & 0 & 0 & 0 & 0 & 0 & 0 & 0 & \frac{-1}{\sqrt{2}} & 0 & 0 & 0 & \frac{1}{\sqrt{2}} & 0 \\
1 & 0 & 0 & 0 & 0 & 0 & 0 & 0 & 0 & 0 & 0 & 0 & 0 & 0 & 0 & 0 & 0 \\
0 & 1 & 0 & 0 & 0 & 0 & 0 & 0 & 0 & 0 & 0 & 0 & 0 & 0 & 0 & 0 & 0 \\
0 & 0 & 1 & 0 & 0 & 0 & 0 & 0 & 0 & 0 & 0 & 0 & 0 & 0 & 0 & 0 & 0 \\
0 & 0 & 0 & 1 & 0 & 0 & 0 & 0 & 0 & 0 & 0 & 0 & 0 & 0 & 0 & 0 & 0 \\
0 & 0 & 0 & 0 & 1 & 0 & 0 & 0 & 0 & 0 & 0 & 0 & 0 & 0 & 0 & 0 & 0 \\
0 & 0 & 0 & 0 & 0 & 1 & 0 & 0 & 0 & 0 & 0 & 0 & 0 & 0 & 0 & 0 & 0 \\
0 & 0 & 0 & 0 & 0 & 0 & 1 & 0 & 0 & 0 & 0 & 0 & 0 & 0 & 0 & 0 & 0 \\
0 & 0 & 0 & 0 & 0 & 0 & 0 & 1 & 0 & 0 & 0 & 0 & 0 & 0 & 0 & 0 & 0 \\
0 & 0 & 0 & 0 & 0 & 0 & 0 & 0 & 1 & 0 & 0 & 0 & 0 & 0 & 0 & 0 & 0 \\
0 & 0 & 0 & 0 & 0 & 0 & 0 & 0 & 0 & 1 & 0 & 0 & 0 & 0 & 0 & 0 & 0 \\
0 & 0 & 0 & 0 & 0 & 0 & 0 & 0 & 0 & 0 & 1 & 0 & 0 & 0 & 0 & 0 & 0 \\
0 & 0 & 0 & 0 & 0 & 0 & 0 & 0 & 0 & 0 & 0 & 1 / 2 & 0 & 0 & 0 & 1 / 2 & 0 \\
0 & 0 & 0 & 0 & 0 & 0 & 0 & 0 & 0 & 0 & 0 & 0 & 1 & 0 & 0 & 0 & 0 \\
0 & 0 & 0 & 0 & 0 & 0 & 0 & 0 & 0 & 0 & 0 & 0 & 0 & 1 & 0 & 0 & 0 \\
0 & 0 & 0 & 0 & 0 & 0 & 0 & 0 & 0 & 0 & 0 & 0 & 0 & 0 & 1 & 0 & 0 \\
0 & 0 & 0 & 0 & 0 & 0 & 0 & 0 & 0 & 0 & 0 & 1 / 2 & 0 & 0 & 0 & 1 / 2 & 0 \\
0 & 0 & 0 & 0 & 0 & 0 & 0 & 0 & 0 & 0 & 0 & 0 & 0 & 0 & 0 & 0 & 1
\end{array}\right], \quad \mathbf{R}=\quad\left[\begin{array}{c}
0 \\
0 \\
0 \\
0 \\
0 \\
0 \\
0 \\
0 \\
0 \\
0 \\
0 \\
0 \\
1 / 2 \\
0 \\
0 \\
0 \\
1 / 2 \\
0 \\
\end{array}\right]
$$

Figure 4: Matrices for the transformation of the LMI variables

The next step should be solving the optimization problem defined in Eq. (54) to obtain a static output-feedback controller

$$
u(t)=G_{y} y(t) .
$$

However, a first attempt of solving this problem with the optimization tools of the MATLAB Robust Optimization Toolbox fails, and the problem is reported to be infeasible. This same difficulty has been encountered in previous works (see $[19,20,28]$ ), and extensive numerical tests indicate that it can be overcome by introducing a small perturbation in the state matrix. More precisely, after replacing the state matrix $\mathbf{A}$ by the perturbed state matrix

$$
\mathbf{A}_{\varepsilon}=\mathbf{A}-\varepsilon \mathbf{I}_{18}
$$

with $\varepsilon=10^{-5}$, the problem in Eq. (54) can be properly solved, resulting the control gain

$$
\tilde{G}_{y}=-6.8719 \times 10^{5}
$$

with an associated $\gamma$-value that satisfies

$$
\gamma_{\tilde{G}_{y}} \leq 0.9782
$$

According to the discussion presented in Remark 2, the actual $\gamma$-value corresponding to $\tilde{G}_{y}$ can be computed by solving the auxiliary optimization problem defined in Eq. (61). Taking the matrix $\hat{\mathbf{G}}$ in Eq. (58) as

$$
\hat{\mathbf{G}}=\tilde{G}_{y} \mathbf{C}_{y},
$$

where $\mathbf{C}_{y}$ is the output matrix given in Eq. (79), we obtain

$$
\gamma_{\tilde{G}_{y}}=0.5083 \text {. }
$$

Moreover, although the gain $\tilde{G}_{y}$ has been computed using the perturbed matrix $\mathbf{A}_{\varepsilon}$, the feasibility of the LMIs in Eqs. (59) and (60) assures the asymptotic stability of the closed-loop matrix $\mathbf{A}_{\tilde{G}_{y}}$ given in Eq. (56).
Remark 7. The procedure presented in Remark 2 can also be applied to compute the energy-to-peak norm of the LQR controller designed in Subsection 4.1.1. By solving the optimization problem defined in Eq. (61) with $\hat{\mathbf{G}}=\tilde{\mathbf{G}}_{I}$, we obtain

$$
\gamma_{\tilde{G}_{I}}=0.4670 \text {. }
$$

Comparing the values presented in Eqs. (74), (85) and (86), we get

$$
\gamma_{\tilde{G}_{I I}}<\gamma_{\tilde{G}_{I}}<\gamma_{\tilde{G}_{y}}
$$

Remark 8. The initial infeasibility of the LMI optimization problems associated to the design of static output-feedback controllers for structural vibration control is certainly a strange phenomenon. Using a perturbed state matrix in the form given in Eq. (81) has proved to be a very effective computational strategy to overcome this problem. Moreover, the method discussed in Remark 2 provides a general procedure to validate the correctness of the controller designed on the basis of the perturbed state matrix $\mathbf{A}_{\varepsilon}$. Currently, we are working on using more general transformations of the LMI variables to provide a better solution to this feasibility problem with promising results.

Remark 9. In all the previous discussions, it has been assumed that $D$ is an ideal active device. In this case, a practical implementation of the output-feedback controller

$$
u(t)=\tilde{G}_{y} y(t)
$$

would only require a velocity sensor allocated in the actuation device $D$, and the control system could be operated using only this local feedback information. From a practical perspective, an even more interesting scenario arises when the actuation device $D$ is considered to be a passive linear damper with adjustable damping capacity $c_{D}$. In this second case, the force exerted by the damper $D$ can be modeled as

$$
f_{D}(t)=-c_{D}\left\{\dot{q}_{4}^{2}(t)-\dot{q}_{4}^{1}(t)\right\}
$$

and the proposed output-feedback controller design strategy provides a systematic procedure to determine the damping capacity 


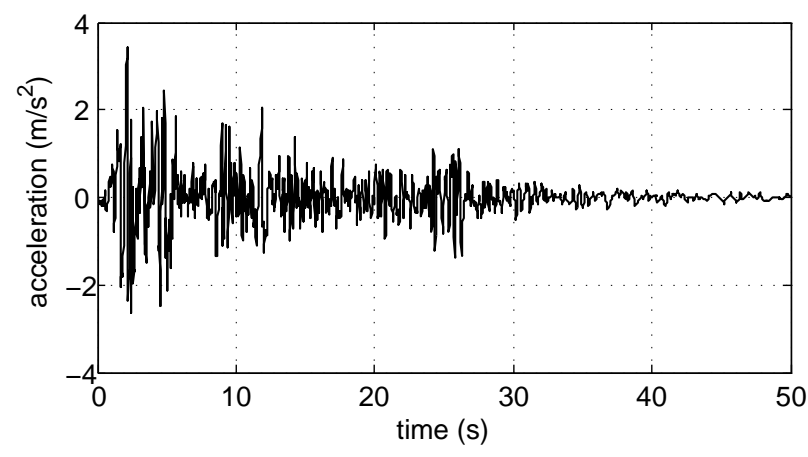

Figure 5: Full scale North-South El Centro 1940 seismic record

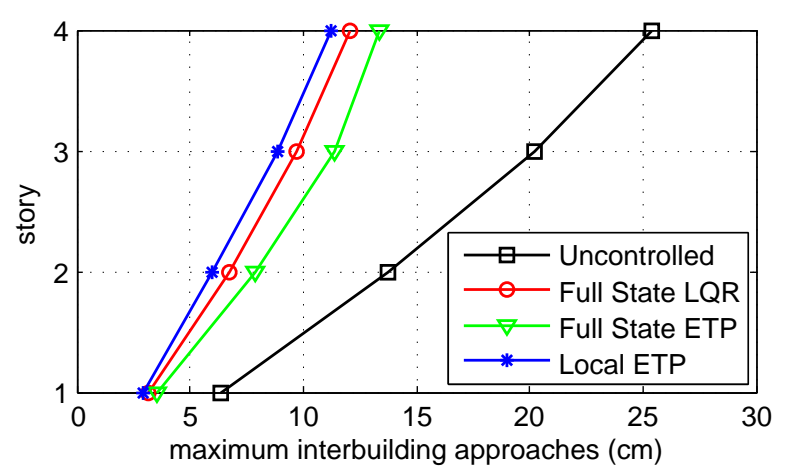

Figure 6: Maximum interbuilding approaches

$c_{D}$. Specifically, for our particular two-building model, we will have

$$
c_{D}=-\tilde{G}_{y}=6.8719 \times 10^{5} \mathrm{Ns} / \mathrm{m} .
$$

A more detailed discussion on the optimal design of passive damping systems for structural vibration control of single buildings using a static output-feedback approach can be found in [20].

\subsection{Numerical simulations}

In this subsection, numerical simulations are conducted to compute the vibrational response of the two-building system for several control configurations. Specifically, the following four control configurations are considered: (1) Uncontrolled. No control system is implemented. (2) Full State LQR. The control system includes an ideal active device $D$, which is driven by the state-feedback LQR controller designed in Subsection 4.1.1. (3) Full State ETP. The control system includes an ideal active device $D$, driven now by the state-feedback energy-to-peak controller designed in Subsection 4.1.2. (4) Local ETP. In this case, we can assume that the control system includes an ideal active device $D$, which is driven by the local velocity-feedback energy-to-peak controller designed in Subsection 4.1.3. Alternatively, and according to Remark 9, we could assume that the actuation device $D$ is a linear passive damper with the damping capacity $c_{D}=-\tilde{G}_{y}$ given in Eq. (90). In all the cases, the full scale North-South El Centro 1940 seismic record is taken

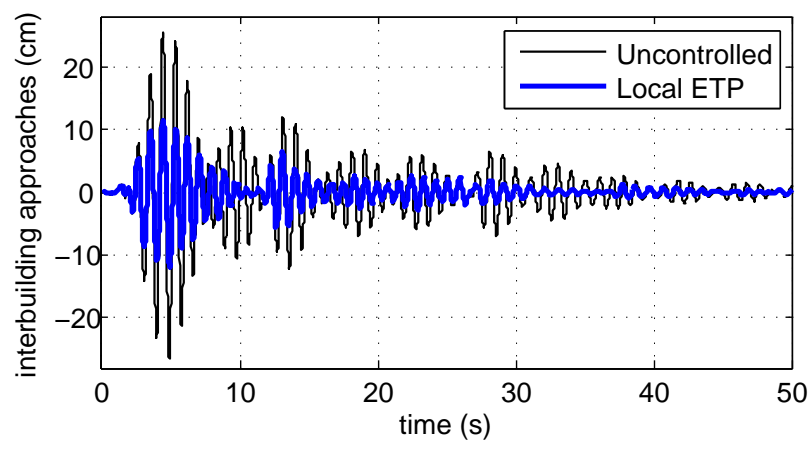

Figure 7: Interbuilding approaches at the fourth story level

Table 1: Percentages of reduction in maximum interbuilding approaches with respect to the uncontrolled response

\begin{tabular}{lcccc}
\hline Story & 1 & 2 & 3 & 4 \\
\hline Full State LQR & 50.8 & 51.0 & 51.9 & 52.5 \\
Full State ETP & 44.5 & 42.7 & 43.7 & 47.5 \\
Local ETP & 54.7 & 56.2 & 56.2 & 55.7 \\
\hline
\end{tabular}

as ground acceleration disturbance (see Fig. 5), and the interbuilding approaches $\mathbf{a}(t)$ together with the interstory drifts $\mathbf{s}(t)$ are computed as output variables. The control effort $u(t)$ is also computed in the controlled cases (2)-(4).

The maximum values of the interbuilding approaches corresponding to the different control configurations are displayed in Fig. 6. A quick inspection of the graphic clearly shows that all the proposed controllers meet the first control design objective of providing a significant protection against pounding events. In particular, interbuilding separations of about $15 \mathrm{~cm}$ can be considered safe for the three controlled configurations while, in contrast, an interbuilding separation of $25 \mathrm{~cm}$ would produce an interbuilding collision for the Uncontrolled configuration. Moreover, it can also be appreciated that the best results are achieved by the Local ETP configuration, which attains levels of reduction in the maximum interbuilding approaches that are uniformly superior to those obtained by the full state configurations. The interbuilding approaches at the fourth story level $a_{4}(t)$ corresponding to the Uncontrolled and the Local ETP configurations are presented in Fig. 7.

The percentages of reduction in maximum interbuilding approaches with respect to the uncontrolled response presented in Table 1 provide a more detailed account of the excellent behavior exhibited by the Local ETP configuration, which achieves percentages of reduction in the interbuilding approaches peakvalues of about $55 \%$ in all the stories. Comparatively, the percentages obtained by the Full State LQR configuration are 3-5 points lower; and this difference is even larger for the Full State ETP configuration, for which the corresponding percentages of reduction are 8-13 points lower.

With regard to the second control design objective of introducing no negative effects in the structural vibration response 


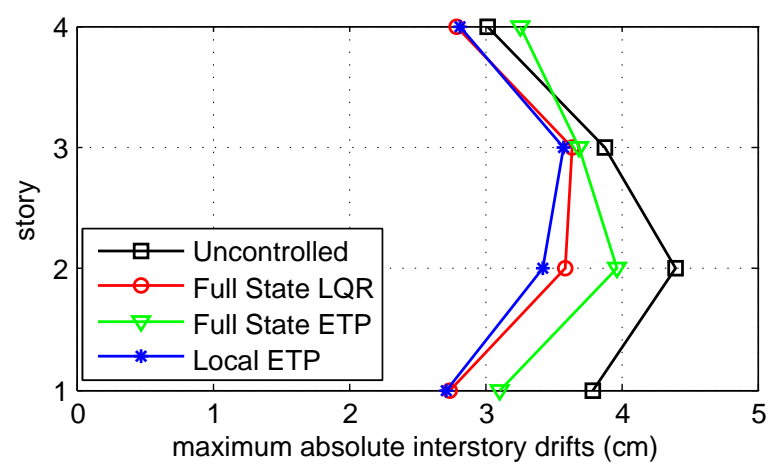

Figure 8: Maximum absolute interstory drifts in buiding 1

Table 2: Percentages of reduction in maximum absolute interstory drifts with respect to the uncontrolled response for building 1

\begin{tabular}{lcccc}
\hline Story & 1 & 2 & 3 & 4 \\
\hline Full State LQR & 27.6 & 18.5 & 6.2 & 7.5 \\
Full State ETP & 17.9 & 9.9 & 5.0 & -8.1 \\
Local ETP & 28.3 & 22.1 & 7.9 & 6.6 \\
\hline
\end{tabular}

of the individual buildings, the graphics of maximum absolute interstory drifts (presented in Fig. 8 and Fig. 9) show that, for building 1, the best results are achieved by the Local ETP configuration. However, the uncontrolled seismic response is slightly exceeded by the response corresponding to the Local ETP configuration for the fifth story of building 2 (see Fig. 9). Something similar happens for building 2 . In this case, the best results are obtained by the Full State ETP configuration, but again the uncontrolled seismic response is also slightly exceeded by the response corresponding to the Full State ETP configuration for the fourth story of building 1 (see Fig. 8). Considering the two-building system as a whole, the best results correspond to the Full State LQR configuration, which attains an appreciable reduction of the interstory drifts peak values for all the stories in both buildings without exceeding in any case the values corresponding to the Uncontrolled configuration. The percentages of reduction in maximum absolute interstory drifts with respect to the uncontrolled response presented in Table 2 and Table 3 provide a more detailed description of the results achieved by the different controlled configurations. The negative values in these tables indicate the cases where the uncontrolled response is exceeded by the response of the corresponding controlled configuration.

An overall consideration of the reduction in interbuilding approaches, the reduction in interstory drifts for both buildings, and the maximum absolute control efforts displayed in Table 4, clearly indicate the excellent performance of the Local ETP configuration which, despite its simplicity, achieves levels of seismic protection similar to those provided by the Full State $L Q R$ configuration, and requiring also similar levels of control effort. However, it must be highlighted the singular characteristics of the Local ETP configuration which, accord-

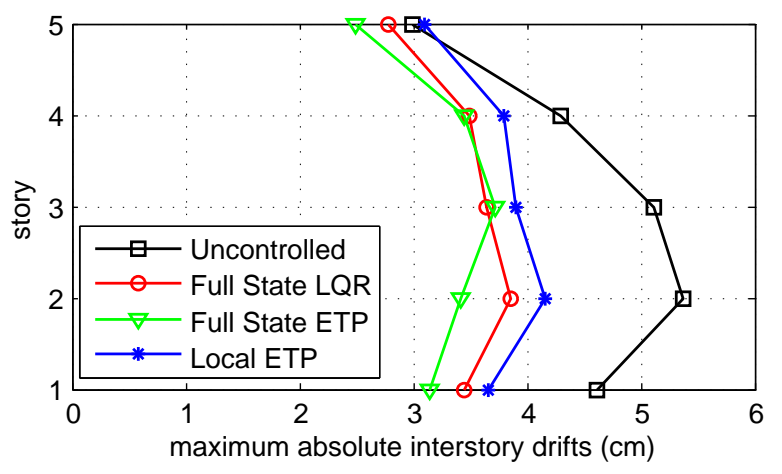

Figure 9: Maximum absolute interstory drifts in building 2

Table 3: Percentages of reduction in maximum absolute interstory drifts with respect to the uncontrolled response for building 2

\begin{tabular}{lccccc}
\hline Story & 1 & 2 & 3 & 4 & 5 \\
\hline Full State LQR & 25.3 & 28.1 & 28.8 & 18.6 & 7.3 \\
Full State ETP & 31.8 & 36.4 & 27.0 & 19.6 & 16.6 \\
Local ETP & 20.8 & 22.5 & 23.7 & 11.4 & -3.7 \\
\hline
\end{tabular}

Table 4: Maximum absolute control efforts (N)

\begin{tabular}{cccc}
\hline Controller & Full State LQR & Full State ETP & Local ETP \\
\hline $\max _{0 \leq t<\infty}|u(t)|$ & $0.64 \times 10^{6}$ & $1.07 \times 10^{6}$ & $0.62 \times 10^{6}$ \\
\hline
\end{tabular}

ing to Remark 9, can be implemented by a passive damper and, consequently, can be operated without sensors, with null power requirements, and no communication system.

Remark 10. Looking at the numerical results presented in this subsection, it becomes quite clear that the Local ETP configuration produces better results than the Full State ETP configuration. It should be noted, however, that these results do not contradict the optimality of the state-feedback energy-to-peak controller computed in Subsection 4.1.2, since the optimality of this controller refers to minimizing the energy-to-peak norm given in Eq. (39), and this fact does not imply a better performance in reducing the interbuilding approach or the interstory drift peak values.

\section{Conclusions and future directions}

In this article, a novel approach to the problem of Structural Vibration Control for multi-structure systems has been presented. The new perspective comprises conceptual, computational, and methodological aspects: Conceptually, the attention is primarily focused on the interactions between adjacent substructures, rather than on the vibrational response of the individual substructures. Computationally, recent advances on static output-feedback control are used to compute simple and 
effective controllers. Methodologically, decentralized velocityfeedback control design strategies are used to design optimal passive-damping systems. To illustrate the main ideas, a control system for the seismic protection of two adjacent buildings linked by a single actuation device has been designed, and numerical simulations have been conducted to assess the effectiveness of the proposed controller with positive results. Although no accurate nor general conclusions can be drawn from such simplified models, the obtained results clearly indicate that the proposed approach is a promising research line that certainly deserves deeper attention and further research effort. In particular, the following four issues are of special interest: (1) Considering more advanced control methodologies to include some practical aspects, such as limited frequency domain [29], actuation saturation [24, 30], or actuation failures $[25,31]$. (2) Extending the study to more complex multistructure systems, which may include three or more adjacent substructures [17]. (3) Considering more complex actuation schemes, which can include interstructure actuation devices together with actuation devices implemented in the individual substructures [16, 17], and also optimal design of passive actuation systems [20]. (4) Providing more general and effective methods to overcome the initial unfeasibility of the optimization problems with Linear Matrix Inequality constraints associated to the design of static output-feedback controllers.

\section{Acknowledgments}

This work was partially supported by the Spanish Ministry of Economy and Competitiveness through the grant DPI201232375, and by the Norwegian Center of Offshore Wind Energy (NORCOWE) under grant 193821/S60 from the Research Council of Norway (RCN). NORCOWE is a consortium with partners from industry and science, hosted by Christian Michelsen Research.

\section{References}

[1] S. Anagnostopoulos, Building pounding re-examined: How serious a problem is it?, in: Proceedings of the 11th World Conference on Earthquake Engineering, paper 2108, Acapulco, Mexico, 1996.

[2] K. Chau, X. Wei, X. Guo, C. Shen, Experimental and theoretical simulations of seismic poundings between two adjacent structures, Earthquake Engineering and Structural Dynamics 32 (2003) 537-554.

[3] P. Komodromos, P. Polycarpou, L. Papaloizou, M. Phocas, Response of seismically isolated buildings considering poundings, Earthquake Engineering and Structural Dynamics 36 (2007) 1605-1622.

[4] D. Lopez-Garcia, T. Soong, Assessment of the separation necessary to prevent seismic pounding between linear structural systems, Probabilistic Engineering Mechanics 24 (2009) 210-223.

[5] P. Polycarpou, P. Komodromos, Earthquake-induced poundings of a seismically isolated building with adjacent structures, Engineering Structures 32 (2010) 1937-1951.

[6] W. Zhang, Y. Xu, Dynamic characteristics and seismic response of adjacent buildings linked by discrete dampers, Earthquake Engineering and Structural Dynamics 28 (1999) 1163-1185.

[7] Y. Ni, J. Ko, Z. Ying, Random seismic response analysis of adjacent buildings coupled with non-linear hysteretic dampers, Journal of Sound and Vibration 246 (2001) 403-417.

[8] Z. Yang, Y. Xu, X. Lu, Experimental seismic study of adjacent buildings with fluid dampers, Journal of Structural Engineering 129 (2003) 197205 .
[9] Z. Ying, Y. Ni, J. Ko, Stochastic optimal coupling-control of adjacent building structures, Computers and Structures 81 (2003) 2775-2787.

[10] A. Bhaskararao, R. Jangid, Seismic response of adjacent buildings connected with friction dampers, Bulletin of Earthquake Engineering 4 (2006) 43-64.

[11] J. Kim, J. Ryu, L. Chung, Seismic performance of structures connected by viscoelastic dampers, Engineering Structures 28 (2006) 183-195.

[12] M. Basili, M. Angelis, Optimal passive control of adjacent structures interconnected with nonlinear hysteretic devices, Journal of Sound and Vibration 301 (2007) 106-125.

[13] R. Christenson, B. Spencer, E. Johnson, Semiactive connected control method for adjacent multidegree-of-freedom buildings, Journal of Engineering Mechanics 133 (2007) 290-298.

[14] S. Bharti, S. Dumne, M. Shrimali, Seismic response analysis of adjacent buildings connected with MR dampers, Engineering Structures 32 (2010) 2122-2133.

[15] H. Zhu, D. Ge, X. Huang, Optimum connecting dampers to reduce the seismic responses of parallel structures, Journal of Sound and Vibration 330 (2011) 1931-1949.

[16] F. Palacios-Quiñonero, J. Rubió-Massegú, J. Rossell, H. Karimi, Semiactive-passive structural vibration control strategy for adjacent structures under seismic excitation, Journal of the Franklin Institute 349 (2012) 3003-3026.

[17] F. Palacios-Quiñonero, J. Rossell, J. Rubió-Massegú, H. Karimi, Structural vibration control for a class of connected multistructure mechanical systems, Mathematical Problems in Engineering, article ID 942910 (2012) 1-23.

[18] J. Rubió-Massegú, J. Rossell, H. Karimi, F. Palacios-Quiñonero, Static output-feedback control under information structure constraints, Automatica 49 (2013) 313-316.

[19] J. Rubió-Massegú, F. Palacios-Quiñonero, J. Rossell, Decentralized static output-feedback $H_{\infty}$ controller design for buildings under seismic excitation, Earthquake Engineering and Structural Dynamics 41 (2012) 1199_ 1205 .

[20] F. Palacios-Quiñonero, J. Rubió-Massegú, J. Rossell, H. Karimi, Optimal passive-damping design using a decentralized velocity-feedback $H_{\infty}$ approach, Modeling, Identification and Control 33 (2012) 87-97.

[21] A. Chopra, Dynamics of Structures. Theory and Applications to Earthquake Engineering, Prentice Hall, Upper Saddle River, New Jersey, USA, 3rd edition, 2007.

[22] N. Kurata, T. Kobori, M. Takahashi, N. Niwa, H. Midorikawa, Actual seismic response controlled building with semi-active damper system, Earthquake Engineering and Structural Dynamics 28 (1999) 1427-1447.

[23] K. Grigoriadis, J. Watson, Reduced order $H_{\infty}$ and $L_{2}-L_{\infty}$ filtering via linear matrix inequalities, IEEE Transactions on Aerospace and Electronic Systems 33 (1997) 1326-1338.

[24] H. Du, J. Lam, Energy-to-peak performance controller design for building via static output feedback under consideration of actuator saturation, Computers and Structures 84 (2006) 2277-2290.

[25] W. Zhang, Y. Chen, H. Gao, Energy-to-peak control for seismic-excited buildings with actuator faults and parameter uncertainties, Journal of Sound and Vibration 330 (2011) 581-602.

[26] MATLAB ${ }^{\mathrm{TM}}$, Control System Toolbox ${ }^{\mathrm{TM}}$ User's Guide, The MathsWorks, Inc., 3 Apple Hill Drive. Natick, MA 01760-20, USA, 2011.

[27] G. Balas, R. Chiang, A. Packard, M. Safonov, MATLAB ${ }^{\mathrm{TM}}$ Robust Control Toolbox ${ }^{\mathrm{TM}}$ 3. User's Guide, The MathsWorks, Inc., 3 Apple Hill Drive. Natick, MA 01760-20, USA, 2011.

[28] J. Rossell, F. Palacios-Quiñonero, J. Rodellar, Semi-decentralized output feedback $H_{\infty}$ control strategy for large building structures, in: Proceedings of the 5th World Conference on Structural Control and Monitoring, paper 112, Shinjuku, Tokyo, 2010.

[29] Y. Chen, W. Zhang, H. Gao, Finite frequency $H_{\infty}$ control for building under earthquake excitation, Mechatronics 20 (2010) 128-142.

[30] H. Du, N. Zhang, F. Naghdy, Actuator saturation control of uncertain structures with input time delay, Journal of Sound and Vibration 330 (2011) 4399-4412.

[31] H. Du, J. Lam, K. Sze, Non-fragile $H_{\infty}$ vibration control for uncertain structural systems, Journal of Sound and Vibration 273 (2004) 10311045. 